\title{
Laser Direct Writing and Photonic Manufacturing of Microball Lens for Wide-angle Imaging, Energy Devices, and Sensors on Flexible Substrates
}

\author{
ShutongWANG ${ }^{1,2}$, Ruozhou $\mathrm{LI}^{2}$, Chong $\mathrm{ZHENG}^{3}$, Cheng $\mathrm{CHENG}^{4}$, Yongchao $\mathrm{YU}^{2}, \mathrm{Shi}_{\mathrm{BAI}}^{3}$, \\ Guoying $\mathrm{FENG}^{1}$, Anming $\mathrm{HU}^{2,3, *}$ \\ ${ }^{1}$ College of Electronics and Information Engineering, Sichuan University, Chengdu 610064,PR China
${ }^{2}$ Department of Mechanical, Aerospace and Biomedical Engineering, University of Tennessee, Knoxville, \\ TN37996, USA \\ ${ }^{3}$ Institute of Laser Engineering, Beijing University of Technology, Beijing 100124, PR China \\ ${ }^{4}$ Department of Electrical Engineering and Computer Science, The University of Tennessee, Knoxville, TN, \\ 37996, USA \\ *Email:ahu3@utk.edu
}

\begin{abstract}
We pursue unique direct writing approaches with femtosecond fiber laser and relevant photonic manufacturing in developing various micro-nano-devices for microelectronics, energy and sensing applications. In this paper, we will present our research progresses on four directions: direct writing of microball lenses for super-wide angle imaging, developing innovative Au/reduce graphene oxide(Au/rGO) super-capacitors with high rates and fast response, multilayer supercapacitors directly written on polyimide and interdigitated capacitance sensors.
\end{abstract}

DOI: 10.2961/jlmn.2016.03.0001

Keywords: laser direct writing, femtosecond laser micromachining, microball lenses, supercapacitors, flexible sensor

\section{Introduction}

Femtosecond laser pulses offer unique characteristics including an ultrashort pulse width and an extremely high peak intensity. ${ }^{[1]}$ These features enable nonlinear lasermatter interactions, such as multiphoton excitation and tunneling ionization. Due to these interactions femtosecond laser becomes a powerful tool for processing of various materials, such as metal, semiconductor, polymer, composite. $^{[2,3]}$

Recently, laser direct writing flexible electronics have shown great promise to enable a variety of novel applications, such as wearable and stretchable sensors, touch screens, energy storage devices, antennas. In comparison with the conventional electronics fabrication techniques, for example photolithography, screen printing and stampimprinting, laser direct writing techniques are facile, highly précised, versatile and especially suitable for inexpensive flexible electronics.

In this paper, we review our latest progress on the micro-nano devices using a femtosecond fiber laser. We began with description of laser direct writing of microball lenses in PMMA with super-wide angle imaging. Next, we discussed fabrication high performance Au/rGO supercapacitors on paper, and flexible super-capacitors on polyimide(PI) sheets. We then focused on interfacial capacitance sensors for Bisphenol A (BPA)detection. Lastly, we summarized and discussed 2D/3D tendencies and the future perspectives.

\section{Femtosecond laser direct writing system}

Fig.1 schematically illustrates a typical laser beam direct writing system. ${ }^{[4]}$ The main components of the system are laser sources, a beam control/shaping system, a microscope objective for producing a tightly focused spot, and a highprecision XYZ translation stage controlled by a computer for $3 \mathrm{D}$ translation of the sample.

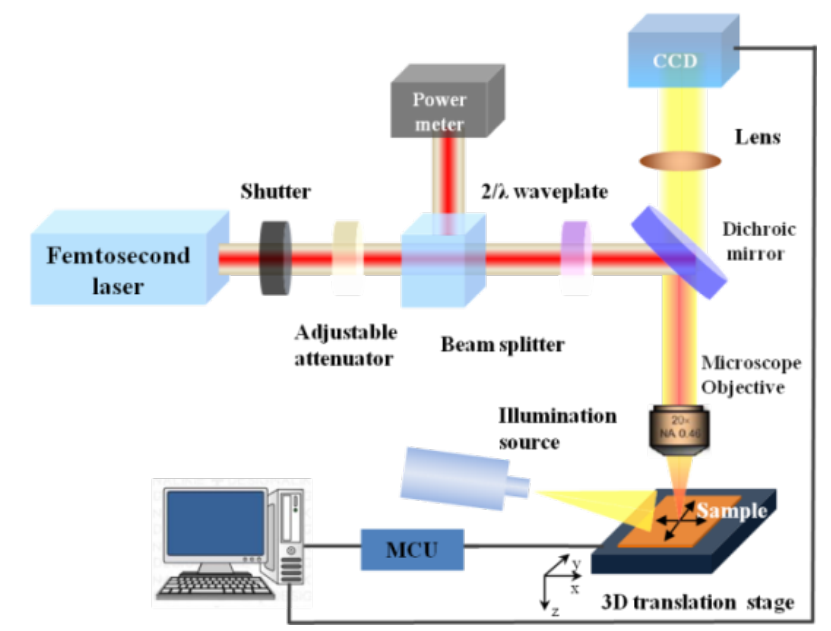

Fig. 1 Skeleton of a 2D/3D laser writing system. The femtosecond laser pulse penetrates serially through the shutter, adjustable attenuator, beam splitter, $2 / \lambda$ waveplate, and an objective lens. The power meter and CCD are used for monitoring the pulse energy and focal state. The sample is translated in the $\mathrm{x}, \mathrm{y}$ and $\mathrm{z}$ directions, and the laser incident direction is along the $\mathrm{Z}$ axis.

The femtosecond (fs) laser pulses generates from a fiber laser system(Cazadero, Calmar Laser Inc.) with a laser quality factor $\mathrm{M}^{2}<1.2,1030 \mathrm{~nm}$ central wavelength, and $400 \mathrm{fs}$ pulse duration repetition rates ranging from $120 \mathrm{kHz}$ to $1 \mathrm{MHz}$. The maximum average power is $2.5 \mathrm{~W}$. The CCD camera and power meter are used to monitor the focal state and the laser energy during the whole writing process. The samples are mounted on a XYZ-translation stage with 
a $500 \mathrm{~nm}$ resolution controlled by a computer. The movement of step-motors was manipulated by a pre-designed computer program written in an Arduino ${ }^{\circledR}$ broad. The program will be translated to the machine language and transfers to an Easydrive broad which connected to the translation stage, thus realizes the 2D/3D pattern directing writing in samples. The Arduino ${ }^{\circledR}$ broad and Easydrive broad constitute the microcontroller unit (MCU). All irradiation was performed in air environment under normal incidence.

A key optical component in this system is the focusing microscope objective because it determines the focal spot size and the fabrication resolution. Another important optical component in the fs laser direct writing system is a tunable attenuator for externally controlling the fs laser power. To ensure stable operation, the output energy of the fs laser will remain constant during fabrication. The pulse energy is varied by either a neutral density variable filters or a halfwave plate placed in front of a polarizer.

\section{Microball lenses for super-wide angle imaging}

Recently, we successfully fabricated two types of embedded microlens function as convex microball lenses (VMBLs) and concave microball lenses (CMBLs) with a fs laser at a repetition rate of $120 \mathrm{kHz}$ irradiated for 5 s which was focused by a $50 \times$ objective.
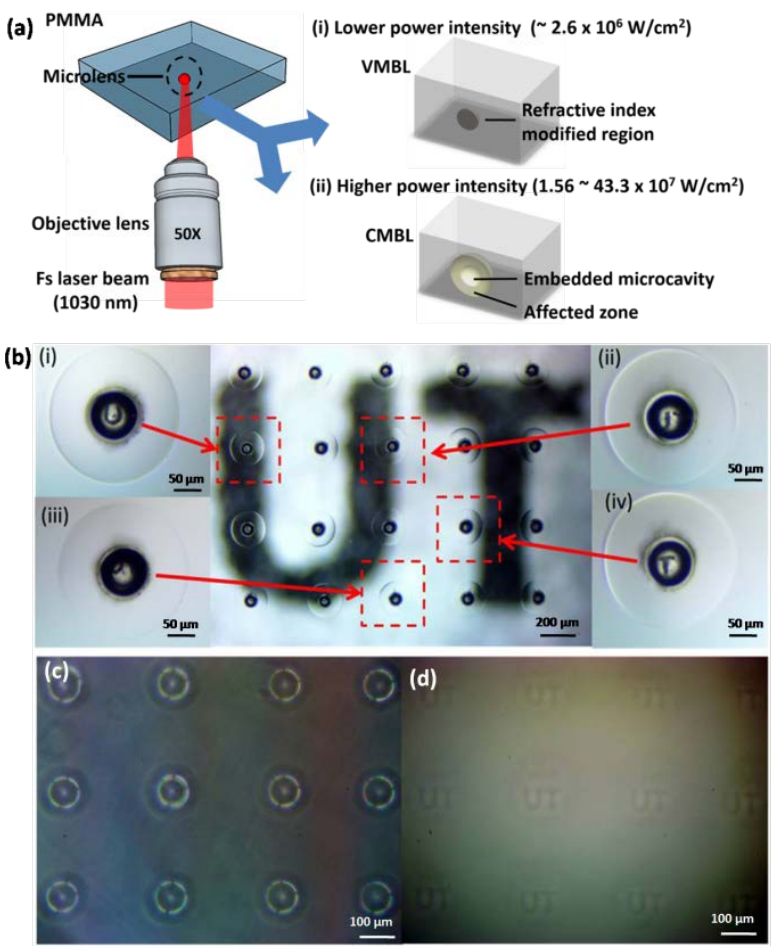

Fig. 2 (a)Experimental layout for the fabrication of two types of embedded VMBL(i) and CMBL(ii).(b) Imaging of the word "UT".(c) Imaging of a lightened ring mask with a $4 \times 3$ VMBLs array. (d) A Letter "UT" is imaged with this VMBL array. ${ }^{[6]}$

The process flow of fabrication mainly consists of the following steps:(1)the PMMA substrate is placed on a 3D translation stage which is located above an objective lens of inverted microscope (Fig.2(a)). (2)The fs laser beam at a repetition rate of $120 \mathrm{kHz}$ is focused inside the PMMA substrate by adjusting the distance of substrate and objective(50×/0.6NA). Different types of microlenses can be acquired by controlling the laser energy with adjusting the ND filter. When the PMMA substrate is irradiated by fs laser pulse with a $90 \mathrm{~mW}$ average power for $5 \mathrm{~s}$, a refractive index modified spherical region is slightly larger than the base PMMA due to the compressive effect of the gas generation at the focal point. This refractive index increased region with the negligible central cavity, as a result, functions as a convex microball lens (VMBL). When the laser power increase to $400 \mathrm{~mW} \sim 1.5 \mathrm{~W}$ laser, the central spherical cavity significantly expanded inside the PMMA substrate. Meanwhile the contribution of the densified region at the surrounding region can be omitted. Such a cavity with a thin densified shell optically functions as a concave microball lens (CMBL) ${ }^{[5]}$. The hollow ball-like cavity of the CMBL is believed generated when the temperature of the irradiated region is heated up to the random scission temperature of PMMA $\left(350{ }^{\circ} \mathrm{C}\right)$ due to the heat accumulation effect of the laser pulse train at a high repetition rate.

To demonstrate the ability of wide angle imaging of the fabricated CMBL array, we used a CMBL array to image the word "UT" which located $0.1 \mathrm{~mm}$ above the CMBL array. The setup is schematically shown in Fig. 2(b). The inset images (i) (iv) in Fig. 2(b) show the selected images taken by different CMBLs. Although the image distortion is especially obvious at the boundary of the cavity, the CMBL array can still work for wide angle imaging. It is important that such a geometrical aberration can be cured by controlled laser writing, which has been developed and published elsewhere. Similarly, a $4 \times 3$ VMBL array is fabricated with a $90 \mathrm{~mW}$ average power at a repetition rate of $120 \mathrm{kHz}$ irradiated for $5 \mathrm{~s}$ per lens. This array is used to image a ring mask (Fig. 2(c)) and a "UT" word (Fig. 2(d)). Although the contrast between the image and the background color is not sharp enough, the shape of the objects can be clearly viewed by CCD. This illustrates that femtosecond laser processing technique is method to fabricate internal microlens in PMMA.

\section{High performance Au/reduce graphene super- capacitors on paper}

With the developing of wearable electronics and information society, integrated energy storage devices on flexible substrates are urgently demanded. Supercapacitors (SCs), which store charge in electrochemical double layers, have a higher charging and discharging speed than batteries, but are still limited by low energy densities and low rate capabilities, due to limited active surface area for charge storage and lower electrode conductivity. Supercapacitors fabricated with carbon nanotubes ${ }^{[7]}$ and graphene ${ }^{[8.9]}$ nanomaterials show the potential for attaining high energy and power densities, but also high intrinsic electrical conductivities. In this part, we will introduce using direct laser-reduction of graphene oxide (GO), as a lithography-free approach, to fabricate in-plane micro-supercapacitors (MSCs) with fast ion diffusion. ${ }^{[10]}$

The fabrication process includes the following steps: dissolving $0.375 \mathrm{mM}$ chloroauric acid $\left(\mathrm{HAuCl}_{4}\right)$ into a 1 $\mathrm{mL}$ GO suspension (7.5 $\left.\mathrm{mg} \mathrm{mL}^{-1}\right)$ ) and then depositing onto a piece of photo paper, direct writing of $\mathrm{rGO} / \mathrm{Au}$ electrodes with laser, removal of unreacted $\mathrm{HAuCl}_{4}$ and applying solid state electrolyte. Fig.3(a) shows the laser writing schematic. Direct laser writing was conducted by either a fs 
laser or a CW laser with $532 \mathrm{~nm}$ wavelength for a comparative study. Laser irradiation causes a strong photothermal effect which not only leads to the decomposition of $\mathrm{AuCl}_{4}{ }^{-1}$ to form $\mathrm{Au}$ nanoparticles, but also promotes the aforementioned reduction reaction of GO. The laser beam was focused with a long working-distance $20 \times$ objective lens $(\mathrm{NA}=0.40)$. By moving the motorized3-axis platform with respect to the focused laser beam, electrode patterns with tunable widths and lengths can be directly written. The MSC contains interdigitated electrodes of approximately $140 \mu \mathrm{m}$ in width with $60 \mu \mathrm{m}$ spacing, conforming to a single cell dimension of $6.9 \mathrm{~mm}$ in length and $1.9 \mathrm{~mm}$ in width. Fig.3(b) shows the morphology of the rGO/Au electrodes. Afterwards, the as-prepared sample is immersed into deionized (DI) water to remove unreacted $\mathrm{HAuCl}_{4}$. After fabricated the electrodes $\mathrm{H}_{2} \mathrm{SO}_{4}$ /PVA hydrogel electrolyte was drop-casted onto the surface $\left(10 \mu \mathrm{Lcm}^{-2}\right)$. Super-capacitor devices were left at room temperature overnight to ensure that the electrolyte completely diffuses into the microelectrodes.

Cyclic voltammetry (CV) examination (Fig. 4a-b) for the electrochemical performance evaluation of laser-written MSCs show that the rGO/Au FS-MSCs(developed with femtosecond laser) provide a faster charge transfer than rGO FS-MSCs (without Au), rGO CW-MSCs (developed with a CW laser), and rGO/Au CW-MSCs. At a low scan rate $\left(1 \mathrm{~V} \mathrm{~s}^{-1}\right)$, these four kinds of super-capacitors all show a good CV curve, but the curve of $\mathrm{rGO} / \mathrm{Au}$ FS-MSCs is much closer to the standard rectangular. Then at a high scan rate $\left(100 \mathrm{~V} \mathrm{~s}^{-1}\right)$, the $\mathrm{rGO} / \mathrm{Au}$ FS-MSCs still retain a quasi-rectangular CV curve, but other two kinds of MSCs with Au NPs diminish to a straight line. From these characterizations, rGO/Au FS-MSCs must have a sufficiently low equivalent series resistance(ESR).

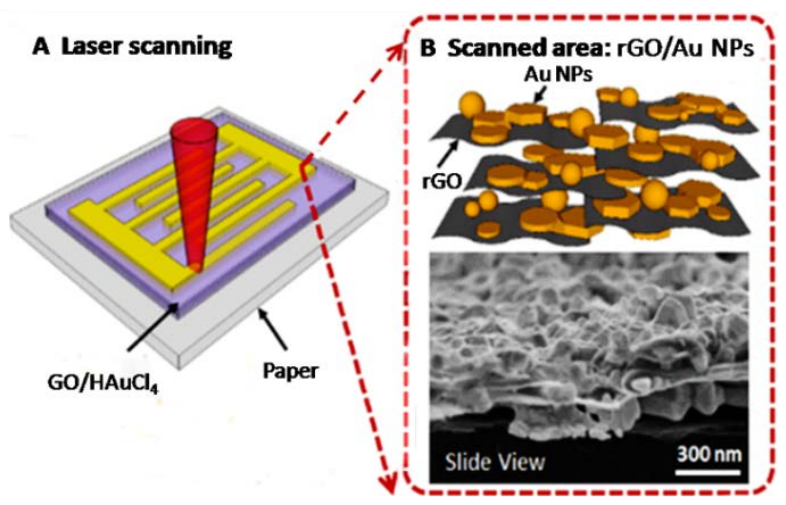

Fig. 3 Schematics of rGO/Au-MSCs (Micro-supercapacitors) fabricated onto a paper substrate. A) Direct FS laser writing of $\mathrm{rGO} / \mathrm{Au}$ microelectrodes, B) the SEM image of rGO/Au microelectrodes. ${ }^{[10]}$

Fig.4(c) show the galvanostatic charge/discharge (GCD) curves for rGO/Au FS-MSCs and rGO FS-MSC. The rGO/Au FS-MSCs show nearly symmetrical triangleshaped curves with no apparent internal resistance drop (iR or voltage drop). However, the curves of rGO FS without Au NPs show about $0.1 \mathrm{~V}$ iR drop, which confirms a relatively larger ESR. For rGO/Au FS-MSCs, the linear dependence of the discharge current density for the scan rate up to $200 \mathrm{~V} \mathrm{~s}^{-1}$ (Fig. 4d) further demonstrates that the use of Au NPs significantly enhances the power output capabilities of MSCs.
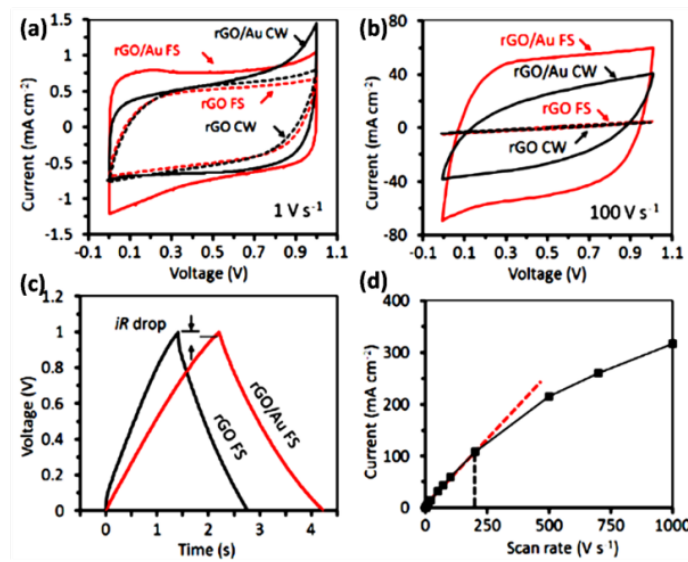

Fig.4 Electrochemical performances of different kinds MSCs: a,b)Cyclic voltammetry curves obtained at scan rates of $1 \mathrm{~V} \mathrm{~s}^{-1}$ and $100 \mathrm{~V}$ $\mathrm{s}^{-1}$, respectively, c) Galvanostatic charge/discharge curves for $\mathrm{rGO} / \mathrm{Au}$ FS MSCs and rGO FS-MSCs measured at $381 \mu \mathrm{A} \mathrm{cm}$, d) the relationship between discharge current and the scan rate for rGO/Au FS-MSCs.

\section{Flexible 3D super-capacitors on polyimide}

Recent studies ${ }^{[11,12]}$ have demonstrated that pulsed laser irradiation rapidly converts the polyimide sheet into an electrically conductive porous carbon structure in ambient conditions. $10 \mathrm{mF} / \mathrm{cm}^{2}$ specific capacitance has been achieved in these single layer surface capacitors. This value now sets the upper limit for electrochemical double layers capacitance for all carbon-based super-capacitors. To further improve the areal capacitance, we propose to using 3D laser direct writing techniques to develop multi-layer super-capacitors.
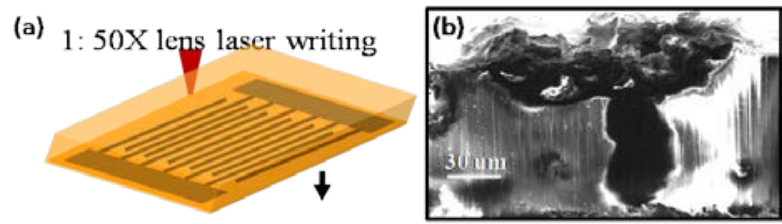

2: $50 \mathrm{X}$ lens laser writing 3: 20X lens laser writing

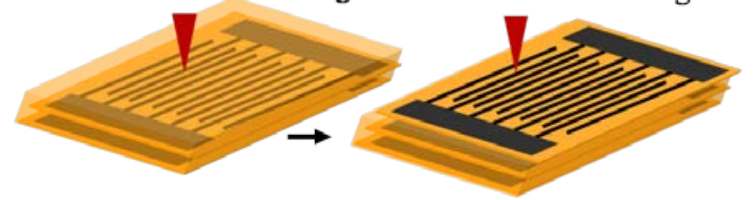

Fig.5(a) Principles for 3D laser direct writing. SEM images of cross section (b) of PI sheets under multi-time scanning.

Fig.5(a) shows the principles for 3D laser direct writing. At first we focused fs laser at the $80 \mu \mathrm{m}$ below the surface of polyimide (PI, thickness $100 \mu \mathrm{m}$ ) sheets. Because of multi-photon absorption polymer is disassociated and result in a carbonization layer with porous structure in the internal of thin PI sheets. Subsequently, we shorten the distance between PI sheets and objective, keeping the focused laser beam still under the surface of PI sheets, then start the second time scanning. Lastly, we shifted the laser focal point onto the surface of PI sheets to carbonize the surface. The cross section after three time scanning is demonstrated in Fig.5(b). After fabricated the electrodes, polyvinyl alcohol-sulfuric acid(PVA- $\left.\mathrm{H}_{2} \mathrm{SO}_{4}\right)$ hydrogel electrolyte was drop-casted onto the surface. Supercapacitor devices were left at room temperature overnight 
to ensure that the electrolyte completely diffuses into the

Fig.6 shows the performance of supercapacitors fabrication with three times scanning on flexible polyimide sheets. Fig. 6(a) shows the galvanostatic charge/discharge (GCD) curves(at the constant current density of 133 $\left.\mu \mathrm{A} / \mathrm{cm}^{2}\right)$ for 3D super-capacitors. The 3D super-capacitors show nearly symmetrical triangle-shaped curves without apparent internal resistance (iR) drop during the chargingdischarging change. Fig. 6(b) shows the specific capacitance curves of 3D super-capacitors at different discharge current densities. The specific capacitance can reach a value of about $37 \mathrm{mF} / \mathrm{cm}^{2}$, which is much higher than the inplane super-capacitors reported in other literatures ${ }^{[11,12]}$. With different focal depths of multi-time scanning we fabricated super-capacitors with controlled 3D structures for enhanced performances. From detailed microstructure characterization we attributed the enhanced conductivity due to laser-induced fusion of graphene-like porous carbon.
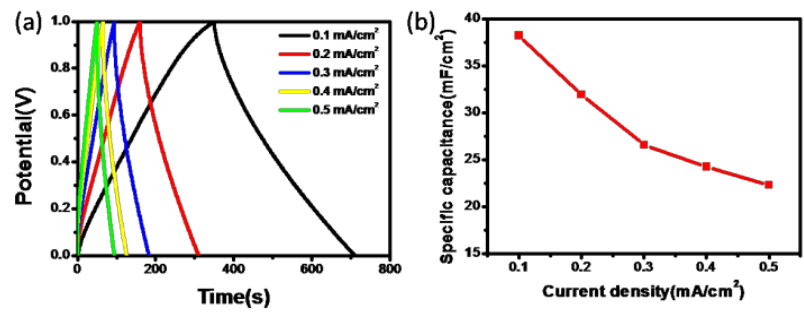

Fig.6 Performance of 3D super-capacitors,(a) galvanostatic charge/discharge responses,(b) specific capacitance calculated from different discharge current rates.

\section{Biomedicine sensors}

In the last few years there had been an explosive increase of the publications on nanomaterial-based biomedical sensing. It is very attractive to fabricate simple, sensitive and stable electronic sensors suited for trace detection in environmental monitoring. Recently, a label-free capacitive sensing method based on alternating current electrothermal(ACET) effect was developed for small molecules (such as Bisphenol A (BPA)) detection, which demonstrated good sensitivity, short response time and simple operation, highly suitable for on-site monitoring. ${ }^{[13]}$

Currently a small sensing area of capacitive BPA sensor limits the absorption volume of BPA molecules, resulting in limited sensing performance and low accuracy. A porous nanostructured surface layer is one of the most effective methods to increasing the absorbed volume of BPA molecules. Conductive circuits and porous structures can be simultaneously realized in simple one-step laser carbonization. As shown in Fig.7(a) interfacial capacitance sensors were fabricated by using a femtosecond laser and a continuous wave (CW) laser to irradiate a commercial flexible polyimide(PI, thickness $100 \mu \mathrm{m})$ sheet under ambient conditions. The dual beams were overlapped and tightly focused onto the sample surface in order to integrate the multiphoton effect from a fs source and the thermal effect from a CW laser. Although the size of focal point is around 3 micrometer based on calculation, the real size of irradiated regions is about $80 \mu \mathrm{m}$ due to the significant thermal effect. The black line in Fig.7(b) illustrates the flexible electrode patterned by the laser, and the light orange re- microelectrodes.

gime indicates pristine PI.Fig.7(c) illustrates the porous flake structures of the irradiated regimes. This porosity is a result of polyimide molecules initially dissociating in the vicinity of focal point of the fs laser as a result of multiphoton absorption. Heating induced by the CW laser brings the polyimide to the carbonization threshold and results in the generation of the porous flake surface morphology.

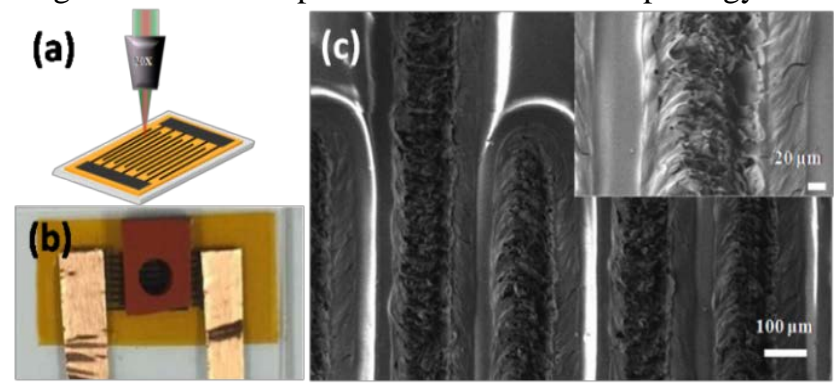

Fig.7(a)Laser direct writing of electrode on a polyimide substrate, (b)Typical photo image of interfacial capacitance sensors,(c) SEM images of the porous carbonized structures.

\section{(a)}
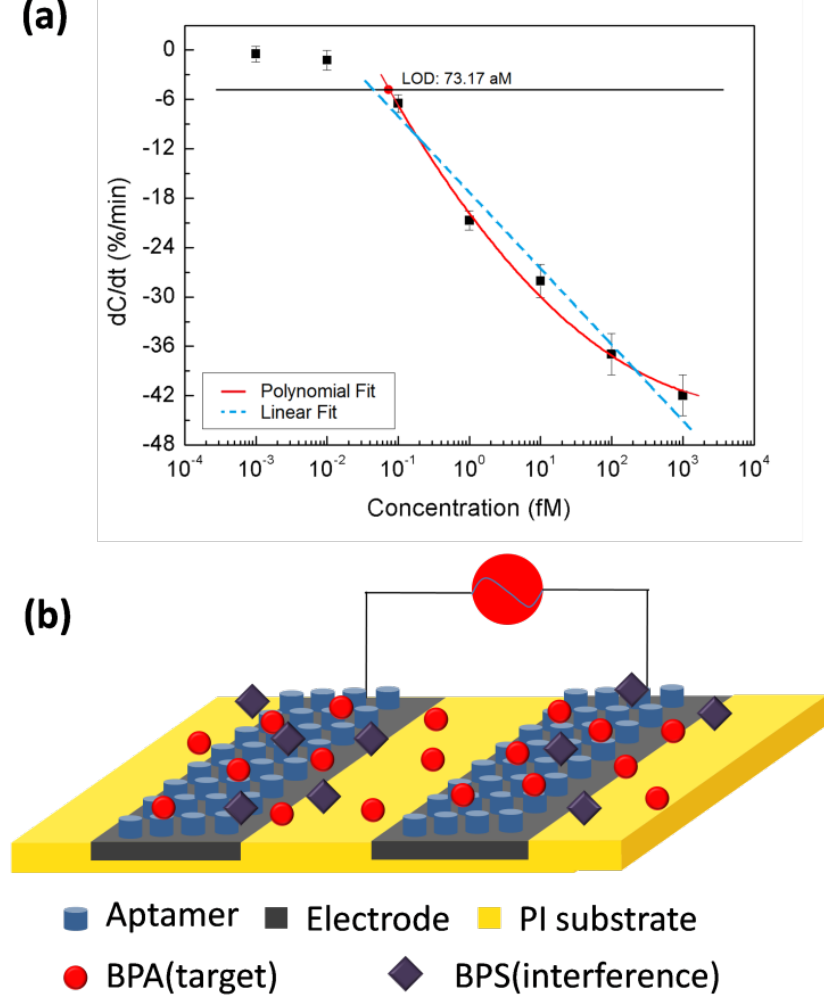

Fig.8(a) Detection response of BPA samples as a function of concentration.(b) Ultrasensitive capacitive sensors with directed movement of complex sample particles with an ACEO effect. ${ }^{[14]}$

Fig.8(a) shows the dose response results of different BPA concentrations ranging from $1 \mathrm{pM}$ down to $0.1 \mathrm{fM}$.The limit of detection was found based on the fitting curve. The cut-off $\mathrm{dC} / \mathrm{dt}$ is found to be $-4.78 \% / \mathrm{min}$, which corresponds to a BPA concentration of73.17aM.Based on AC electromosis(AECO) effect, BPA particles are carried to the electrode's surface by fluid flows during capacitance measurement, together with interference particles such as $\mathrm{Bi}$ sphenol S (BPS). Since the aptamer deposited on the electrode's surface can only bind with BPA specifically, trace concentrations of BPA increase the layer thickness and de- 
crease the interfacial capacitance. The nanostructured surface increases the area to immobilize aptamer, significantly increasing the sensitivity. Fig.8(b) summarized the sensing mechanism.

\section{Summary and future outlook}

To summarize, various devices have been successfully fabricated by femtosecond laser direct writing. An CMBL array is fabricated on PMMA substrates for wide angle imaging. In-plane reduced graphene/Au micro-super-capacitors (MSCs) demonstrate a high rate performance with fast ion diffusion. To further improve the performance(power density), we have fabricated flexible multilayer super-capacitor based on polyimide sheets. This kind of super-capacitor can be also used as a interfacial capacitance sensor to detect BPA at a limit of detection of $73.17 \mathrm{aM}$.

The future development of this research field will benefit wearable energy and portable sensing devices. With future development of precise laser manufacturing in 3D, energy devices with a high energy storage density are expected. Flexible sensor devices with 3D architecture are also promising for multi-functional sensing by laser multilayer writing. Besides, laser writing will target a mass production, a crucial step for commercialization.

\section{Acknowledgements}

We appreciate the research initiative funding provided by the University of Tennessee as a new hire package to $\mathrm{AH}$. This work is also partially supported by National Natural Science Foundation of China (51575016), the Beijing Oversea High-Level Talent Project and a strategic research grant(KZ20141000500, B-type) of Beijing Natural Science Foundation P. R. China. This work is supported in part (S. Wang) by the scholarship from China Scholarship Council (CSC) under the Grant CSC NO.201506240097.

(Received: May 23, 2016, Accepted: August 1, 2016)

\section{References}

[1] A. Hu, M. Rybachuk, Q.-B. Lu, W. W. Duley, Appl. Phys. Lett. 91 (2007) 131906

[2] A. Y. Vorobyev and C. Guo, Applied Physics Letters 92, (2008)041914.

[3] S. Sundaram and E. Mazur, Nature materials. 1, (2002)217.

[4] K. Sugioka and Y. Cheng, Advanced Optical Technologies 1, (2012)353.

[5]C. Zheng, A. Hu, K. D. Kihm, Q. Ma, R. Li, T. Chen, and W. Duley, small 11, (2015)3007.

[6] C. Zheng, A. Hu, R. Li, D. Bridges, and T. Chen, Optics Express 23, (2015)17584.

[7] D. N. Futaba, K. Hata, T. Yamada, T. Hiraoka, Y. Hayamizu, Y. Kakudate, O. Tanaike, H. Hatori, M. Yumura, and S. Iijima, Nature materials 5, (2006)987.

[8] Z. S. Wu, K. Parvez, X. Feng, and K. Müllen, Nature communications 4 (2013),2487.

[9] M. F. El-Kady and R. B. Kaner, Nature communications 4, (2013)1475.

[10] R.-Z. Li, R. Peng, K. Kihm, S. Bai, D. Bridges, U. Tumuluri, Z. Wu, T. Zhang, G. Compagnini, and Z. Feng, Energy \& Environmental Science 9 (2016) 1458.

[11] J. B. In, B. Hsia, J.-H. Yoo, S. Hyun, C. Carraro, R. Maboudian, and C. P. Grigoropoulos, Carbon 83, (2015)144

[12] J. Lin, Z. Peng, Y. Liu, F. Ruiz-Zepeda, R. Ye, E. L. Samuel, M. J. Yacaman, B. I. Yakobson, and J. M. Tour, Nature communications 5 (2014) 5714.

[13] H. Cui, C. Cheng, X. Lin, J. Wu, J. Chen, S. Eda, and Q. Yuan, Sensors and Actuators B: Chemical 226, (2016) 245

[14] C. Cheng, S. Wang, J. Wu, Y. Yu, R. Li, S. Eda, J. Chen, G. Feng, B. Lawrie, and A. Hu,ACS Appl. Mater. Interfaces,(2016). DOI: 10.1021/acsami.6b03743. 